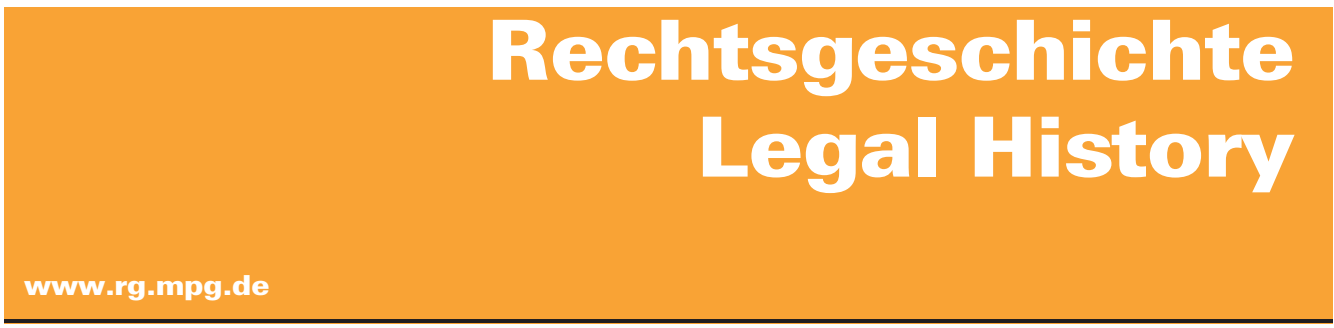

http://www.rg-rechtsgeschichte.de/rg24

$\mathrm{Rg} 22016$

$510-513$

Zitiervorschlag: Rechtsgeschichte - Legal History Rg 24 (2016)

http://dx.doi.org/10.12946/rg24/510-513

\title{
Helge Dedek
}

\section{Privat-Justiz}


auch in Hinblick auf die Frage, inwiefern und von wem Recht als diskursiver Referenzrahmen "gesprochen" oder aber, im Sinne Ciceros, zum Schweigen gebracht wird. Hierfür liefert insbesondere Isabell V. Hull mit ihrem neuen Buch auch methodisch wichtige Impulse. Einer Auseinandersetzung mit Recht und Krieg im Sinne einer interdisziplinären Völkerrechtsgeschichte als kritische
Geschichte der Völkerrechtspolitik, die die Multinormativität internationaler Beziehungen zwischen Konstitutionalisierung und Fragmentierung in historischer Perspektive ernstnimmt, sind beide Bände, der eine als Einführung, der andere als innovative Detailstudie, sehr angemessen.

\section{Helge Dedek Privat-Justiz*}

2005, besetztes Bagdad, „Green Zone«, die schwer befestigte amerikanische Sicherheitszone. Unheil wirft seine Schatten voraus: »[T]he Green Zone never became the safe haven that it was supposed to be for a twenty-year-old Texan named Jamie Leigh Jones« (4). In der Tat wird sich Furchtbares ereignen. Jones, zivile Angestellte einer Tochter des Halliburton-Konzerns, wird von mehreren Männern, ebenfalls in den Diensten Halliburtons, vergewaltigt, misshandelt und schwer verletzt. Damit nicht genug: Nach einer ärztlichen Untersuchung wird Jones in einer Baracke eingesperrt und es wird ihr jeder Kontakt zu ihren Angehörigen untersagt. Einer der Wachsoldaten erbarmt sich, Jones kann ihren Vater kontaktieren, der über politische Kanäle erreicht, dass man Jones in die USA zurückreisen lässt. Heimgekehrt, hofft Jones auf Genugtuung durch die Justiz. Doch strafrechtliche Verfolgung bleibt aus. Jones erhebt Zivilklage gegen ihren Arbeitgeber, der nicht nur den unglaublichen Freiheitsentzug angeordnet, sondern zudem abredewidrig keinerlei Sicherheitsmaßnahmen für weibliche Angestellte getroffen hatte. Jedoch: »[T]he courthouse door of the civil system was already closed « (5) - der Weg zu einem staatlichen Zivilgericht war wegen einer Schiedsklausel im Arbeitsvertrag (zumindest teilweise) versperrt.

Mit dieser Fallschilderung ${ }^{1}$ beginnt Imre Szalai sein Buch über die Geschichte des Schiedsverfah- rensrechts in den USA. Der proverbiale »day in court « ist in den USA einer der Kristallisationspunkte der kollektiven Imagination - in einer extrem stratifizierten Gesellschaft ist es einer der mächtigsten amerikanischen Heilsmythen, dass David vor Gericht Goliath in die Knie zwingen könne, Stoff zahlloser Erzählungen und vor allem Filme, »court room dramas«, die mit diesem Topos spielen. Die filmische Dramaturgie der Eingangsszene funktioniert daher auf verschiedenen Ebenen, versetzt den Leser einerseits hinein in eine "Handlung« und ist andererseits in ihrer Bildhaftigkeit selbst emblematisch: Film ist das natürliche Medium zur Inszenierung des »day in court«, seinerseits charakterisiert durch das Rechtsschauspiel vor der Kamera. Die Form des bildmedialen Genrezitats steigert noch das Bewusstsein um das, was hier versagt wird.

So trifft die Eingangsszene und der ihr zugrundeliegende unfassbare Sachverhalt den Leser wie ein Faustschlag. Einer solchen Überwältigung hätte es wahrscheinlich gar nicht bedurft, zumindest den im Sozial- oder Wohlfahrtsstaat europäischen Zuschnitts sozialisierten Leser zu gewinnen: Dass selbst in formularmäßigen Verbraucher- und Arbeitsverträgen Schiedsvereinbarungen allenthalben anzutreffen und für wirksam gehalten werden, scheint ein schwer nachvollziehbarer Aspekt amerikanischen »Exzeptionalismus ${ }^{2}{ }^{2}$ In den USA ist
* Imre S. Szalai, Outsourcing Justice. The Rise of Modern Arbitration Laws in America, Durham, NC: Carolina Academic Press 2013, xii, 284 S., ISBN 978-1-61163-202-6
1 Jones v. Halliburton, 583 F.3d 228 (5th Cir. 2009), aff'd 9625 F. Supp. 2d 339 (S.D. Tex. 2008).

2 Siehe z. B. Амy J. Sснмiтz, American Exceptionalism in Consumer Arbi- tration, 10 Loy. U. Chi. Int'l L. Rev. 81 (2012). 
diese gängige Praxis jedoch Gegenstand einer rechtspolitischen Debatte, die sich rhetorisch kaum Restriktionen auferlegt. Szalai ist gerade im Hinblick auf die Wahl seiner Einleitung bloße Effekthascherei vorgeworfen worden; sein Buch falle in eine Literaturgattung, in der "poseurs and ideologues" gegen die Arbitrage Stimmung machten. Befeuert sei all dies letztlich von der ungezügelten Gier der "so-called plaintiffs' bar", die sich mit den laienbesetzten Juries leichteres Spiel erhoffe, als mit einem mit »Experten « besetzten Schiedsgericht. ${ }^{3}$ Keinen Hehl macht zum anderen auch Szalai aus seinen konkreten rechtspolitischen Beweggründen und Zielen. Er beschreibt die Kampfzone von der anderen Seite der Front: Sein Forschungsinteresse sei geweckt worden, als er in seiner Anwaltstätigkeit die fatalen Auswirkungen der Schiedsklauseln unmittelbar erlebt habe, mit der Großkonzerne strukturell unterlegene Vertrags-»Partner « vorsätzlich und systematisch von den Gerichten - und damit dem "pursuit of justice» - fernhielten. Sein Buch widmet er folgerichtig »the Millions of Americans Who Are Unjustly Bound by an Arbitration Agreement« (v). Die Debatte schlägt auch jenseits der Fachjournale Wellen; die New York Times etwa widmete unlängst den Effekten der Schiedsklauseln, aber auch der gezielten Lobbyarbeit, denen sich deren endemische Verwendung verdankt, eine Artikelserie, in der schon die Titel der Beiträge in Ton und Tendenz ganz klingen wie bei Szalai: "Beware of the Fine Print, Part I - Arbitration Everywhere: Stacking the Deck of Justice ${ }^{4}$ etwa, und »Beware of the Fine Print Part II - In Arbitration, a >Privatization of the Justice System. « ${ }^{5}$ Die Botschaft ist klar: Die exzessive Justizprivatisierung ist ein abgekartetes Spiel, bei dem die schwächere Partei immer verliert.

Wie kommen wir von dieser handfesten Auseinandersetzung zur Rechtsgeschichte? Szalai, wie andere Autoren auch, ${ }^{\mathbf{6}}$ will der Geschichte Munition abgewinnen zur Bekämpfung des konzertiert ausgedehnten Einsatzes der Schiedsklauseln. Die Gedankenführung ist schlicht und läuft in letzter
Konsequenz auf ein einfach verstandenes "originalistisches« Argument hinaus: Die Gesetzgebungsakte, auf die Gerichte heute die Sanktionierung der Praxis stützten, hätten eine solche Anwendung nie beabsichtigt, daher sei die gegenwärtig gängige Auslegung fehlsam (191 ff.). Seine Beschreibung von Jones $v$. Halliburton schließt Szalai: »The law was never intended to apply in the employment context, and courts in the United States have repeatedly and grossly misinterpreted this law « (6); und diese "Fehlinterpretation « ist es, die die bereits eingangs beschworenen Türen der Gerichtssäle zuschlug, und zwar wiederum für »millions of Americans« (10).

"This law», das ist in Szalais Untersuchung vor allem der Federal Arbitration Act von 1924. Die Rechtsgeschichte, die Szalai erzählt, behandelt vornehmlich die von der New Yorker Chamber of Commerce betriebene Kampagne, gesetzliche Grundlagen für ein kaufmännisches Schiedsgerichtswesen zu schaffen. Szalai stellt den von ihm als energetischen Antreiber geschilderten Charles Leopold Bernheimer, "Father of Commercial Arbitration" (25), in den Mittelpunkt seiner Darstellung. Szalai wertet durchaus neue Quellen aus, vor allem auch, um dem Bild von Bernheimer weitere biographische Details hinzuzufügen. Das mündet dann etwa in dem Versuch, Bernheimers Begeisterung für die außergerichtliche Streitbeilegung mit dessen friedfertigem Charakter und ausgleichendem Wesen in Verbindung zu bringen (26). Szalai beschreibt detailliert, wie die Kampagne der Handelskammer immer weitere Kreise zog und zuletzt auf nationaler Ebene reüssierte ( $97 \mathrm{ff}$.). Szalai bringt auch neues Material zum Gesetzgebungsprozess selbst, etwa zu den Anhörungen im Kongress 1923 (135f.) und 1924 (160).

Szalai koloriert hier mit vielen Details und Beobachtungen eine im Wesentlichen bekannte Geschichte. Spannender wird es, wenn Szalai den Versuch unternimmt, diese Vorgänge in den Kontext der progressiven politischen Bewegung zu Beginn des Jahrhunderts und der "progressive procedural reform« zu stellen. Dabei betritt Szalai
3 William W. Park, Arbitration's Discontents, in: Mélanges en l'honneur du Professeur Bernard Audit: Les relations privées internationals, Paris 2014, 581.

4 New York Times vom 1. November 2015, A1.
5 New York Times vom 2. November 2015, A1.

6 Siehe etwa Katherine Van Wezel Stone, Rustic Justice: Community and Coercion under the Federal Arbitration Act, 77 N.C.L. Rev. 931 (1999) 979 ff.; zuletzt Hiro N.
Aragaki, The Federal Arbitration Act as Procedural Reform, 89 N.Y.U. L. Rev. (2014) 1939 ff. 
Neuland. Sein Verständnis des "progressivism« verlässt sich allerdings in der Hauptsache auf das Buch Robert Wiebes von 1967, ${ }^{7}$ das er über Seiten hinweg paraphrasiert (174 ff.). Wiebe und der von Szalai nicht in Bezug genommene Samuel Hays ${ }^{8}$ stehen für den an Weber orientierten institutionellen oder »organisationellen «" Ansatz zur Interpretation der Progressive Era. Progressivismus wird so als »rebellion against limited government and the individualism of nineteenth-century liberalism « (Larry Walker) ${ }^{\mathbf{1 0}}$ gelesen, in der ein aufstrebender Mittelstand seine Hoffnung auf den modernen Verwaltungsstaat, auf Professionalisierung und Bürokratisierung der Strukturen setzt. Diesen Interpretationsrahmen für die Privatisierung von Justizfunktionen fruchtbar zu machen, scheint auf den ersten Blick kontraintuitiv. Doch Szalais Deutung der arbitration in diesem Kontext als "modernes « Modell effizienter, pathosbefreiter Streitbeilegung durch »Experten « überzeugt; zumal, wenn man zwecks Kontrastierung als Vergleichsbild den Richter des common law des 19. Jahrhunderts hinzudenkt, der, mit weitem Ermessen mittelalterliche Formen und Begriffe handhabend, als eine Art Gentleman-Rechtsorakel das exakte Gegenbild zum Weberschen Rationalisierungsideal verkörpert.

Dieser Gedanke hätte nun Ausgangspunkt für weitere Fragestellungen sein können. Etwa: Wird der Interpretationsschlüssel dem Reformimpetus hinreichend gerecht, gibt es Kehrseiten der "progressiven «Verfahrensrechtsrefom, die auch für das Schiedsverfahren Bedeutung erlangen? Der Ansatz einer kritischen Lesart der Reformbestrebungen unter Berufung auf Gabriel Kolko - der zur selben Zeit wie Wiebe und Hays den Regulierungseifer der Progressive Era provokativ als Aktionismus konservativer Besitzstandwahrung erklärte - wird im Keim erstickt, indem die Komplexität des Phänomens eigenartig kurzschlüssig wieder auf Bernheimers persönliche Intentionen reduziert wird. Dessen »mindset « habe eher in Einklang mit Wiebes Erklärungen gestanden, idealistisch (und eben nicht »zynisch«) und guten Willens habe Bernheimer an den Fortschritt durch Reform geglaubt (178).

Das ist freilich methodisch wie inhaltlich unbefriedigend. So bleibt Szalai auch insgesamt seltsam farblos; dies gilt trotz der Detailfülle. $\mathrm{Zu}$ oft werden nur Klischees bedient, wie etwa bei dem Versuch, für den Leser die Reformen der zwanziger Jahre sozialgeschichtlich zu kontextualisieren: "The Roaring Twenties, the Jazz Age, the New Era, Prohibition, Al Capone, The Great Gatsby, the Charleston, Lindbergh's flight, flappers, Babe Ruth and the Yankees, Jack Dempsey, Knute Rockne and the Four Horsemen of Notre Dame, the Scopes Trial, Sacco and Vanzetti, stalking movies`, Greta Garbo, Walt Disney and Mickey Mouse, and the rise of advertising, consumerism, commercial radio, and automobiles. The decade of the 1920s was a period of $>$ dramatic social, economic, and political change « (97). Anstelle einer Beschreibung von gesellschaftlichem Wandel soll hier das atemlose Staccato der Bilderfetzen amerikanischer Popkultur den Leser den »Zeitgeist" viszeral erleben lassen. Gegen diese wie andere dramaturgische Techniken soll nun nicht eingewandt werden, dergleichen habe in wissenschaftlichen Werken nichts zu suchen - nur bietet Szalai einfach kein großes Kino. Das Kolportagehafte, die Abgeschmacktheit der Bilder, die mangelnde Berührungsangst gegenüber übergroßem Pathos, die allgemeine stilistische Unbeholfenheit: Das alles wirkt selbst auf den um Sympathie mit der Tendenz des Unternehmens bemühten Leser ermüdend und auch befremdlich. Dieses ist aber kein rein ästhetisches Monitum und (hoffentlich) kein Kulturdünkel; Szalai verschließt sich mit seinen klischeeverliebten Erklärungsmustern und dem zu einfachen Schema einer großen amerikanischen Errungenschaft, die in der Folge tragisch missverstanden wird, von vornherein den Zugang zu einer komplexeren Analyse.

Besser verständlich wird diese Vorgehensweise, wenn man sie als genrespezifisch begreift. Es geht nun einmal letztlich um einen Beitrag zu einer in
7 Robert H. Wiebe, In Search for Order, 1887-1920, New York 1967.

8 Siehe etwa Samuel P. Hays, Political Parties and the Community-Society Continuum, in: Walter D.

Burnham, William N. Chambers (Hg.), The American Party Systems, New York 1967, 152 ff.; Samuel P.
Hays, The Social Analysis of American Political History, 1880-1920, Political Science Quarterly 80 (1965) $373 \mathrm{ff}$.

9 Siehe etwa Louis Galambos, The Emerging Organizational Synthesis in Modern American History, Business History Review 44 (1970) 279 ff.;
Wayne K. Hobson, Professionals, Progressives and Bureaucratization: A Reassessment, in: The Historian 39 (1977) $639 \mathrm{ff}$.

10 Larry Walker, Woodrow Wilson, Progressive Reform, and Public Administration, in: Political Science Quarterly 104 (1989) 509. 
der medialen Öffentlichkeit aggressiv geführten (rechts-)politischen Debatte; und Szalai spielt (bewusst oder unbewusst) nach den Regeln des politischen Diskurses im amerikanischen mainstream. So hält sich Szalai den Vorwurf des Zynismus und den Ruch, zu weit links zu stehen - was der Salonfähigkeit seiner Argumente Abbruch täte - tunlichst vom Leibe. Auf dem Umschlag des Buches ist das Foto einer Portion Pommes Frites zu sehen, in einer in Fastfood-Ketten üblichen Pappverpackung, auf der statt eines Markenlogos aber hier Stars \& Stripes prangen. Ein, so will es scheinen, ironischer Kommentar zu eben dem amerikanischen Konsumerismus und der Niedriglohnkultur, in der der von Szalai angeprangerte Wildwuchs am prächtigsten gedeiht. Wie Szalai auf seiner Internet-Seite erklärt - wo er zuvörderst verdienstvoll über jüngste Entwicklungen im Schiedsrecht informiert -, ist dieses Verständnis durchaus bezweckt; zumal stößt man dort auf den Hinweis ${ }^{\mathbf{1 1}}$ (Vordergründigkeit verhindert Missverständnisse - ein Misstrauen gegenüber allem Subtilen, das das ganze Buch durchzieht), dass sich an der Unterkante der Pappschachtel eine mikroskopisch gedruckte Schiedsklausel findet (die man in der Tat beim zweiten Hinsehen ausmachen kann). Wenn aber Szalai stets den amerikanischen Entrepreneursgeist seiner Protagonisten hervorhebt und im Vorwort artig beteuert, bei aller Kritik am Supreme Court nie seine Dankbarkeit für das Privileg amerikanischer
Freiheit aus den Augen verloren zu haben: ein Hautgout von freedom fries.

Vorbehalte, derer viele sich angesichts all dessen nicht werden erwehren können, dürfen indes nicht vergessen machen, dass Szalais Buch einen - gerade durch seinen Stil und seine Methode effektiven (i.e. häufig zitierten) Beitrag zu einer Debatte geliefert hat, die die wichtige öffentliche Auseinandersetzung mit einer offensichtlich perversen Praxis befördert. In dieser Dimension, nicht primär als ein eigentümlich instrumentelles rechtshistorisches Projekt, sollte man es bewerten. Es ist ein unironisches und unverblümtes, wenn man so will, in seiner Plakativität ehrliches Buch: Man glaubt Szalai ohne Weiteres, dass es ihm wie seiner Hauptfigur Bernheimer (wenn man Szalais Schilderung folgt) ohne unlautere Motive um die Sache geht. Ihm kommt überdies das große Verdienst zu, mit dem Eifer seiner Darstellung Neugier just auch an einer weiteren historischen Befassung mit den Ursprüngen der arbitration in den USA geweckt zu haben. Amalia Kessler etwa hat jüngst vorgemacht, ${ }^{12}$ wie man, durchaus auf den Vorarbeiten Szalais aufbauend, sich der progressiven Prozessrechtsreform in ihrer ganzen Vielschichtigkeit und Ambiguität nähern und so zu dem Thema kritische, differenzierte und theoretisch informierte Rechtsgeschichte betreiben kann.

11 http://www.outsourcingjustice.com/ french-fries/

12 Amalia D. Kessler, Arbitration and Americanization: The Paternalism of Progressive Reform, 124 Yale L.J. 2015, $2940 \mathrm{ff}$ 